\title{
水稻内源油菜素甾醇对施氮量的响应及其对颖花退化的调控作用
}

\author{
姚佳瑜 1,2 于吉祥 1,2 王志琴 1,2 刘立军 ${ }^{1,2}$ 周 娟 ${ }^{1,2}$ 张伟杨 $1,2,{ }^{*}$ \\ 杨建昌 $1,2, *$
}

${ }^{1}$ 江苏省作物遗传生理重点实验室 / 江苏省作物栽培生理重点实验室 / 扬州大学农学院, 江苏扬州 $225009 ;{ }^{2}$ 江苏省粮食作物现代产 业技术协同创新中心 / 扬州大学, 江苏扬州 225009

摘 要: 为探明油菜素甾醇(brassinosteroids, BRs)是否介导氮肥对水稻颖花退化的影响。水稻品种扬稻 6 号和角优 2640 种植于盆钵, 全生育期设置 3 种施氮水平, 观察了不同氮肥处理下水稻减数分裂期幼穗中氮含量、BRs、过氧化 氢 $\left(\mathrm{H}_{2} \mathrm{O}_{2}\right)$ 和总抗氧化能力(total antioxidant capacity, T-AOC)水平及其与颖花退化的关系。结果表明, 颖花退化率的降 低与稻穗中增加的 24-表油菜素甾酮 (24-epicastasterone, 24-epiCS)和 28-高油菜素内酯 (28-homobrassinolide, 28-homBL) 含量密切相关。当稻穗的氮含量为 $1.25 \%$ 时, 幼穗中 BRs (24-epiCS 和 28-homBL)含量显著增加, 颖花退 化率显著降低。稻穗中 T-AOC 水平与 BRs 含量变化趋势相同, 且均与水稻颖花退化率显著负相关, 而 $\mathrm{H}_{2} \mathrm{O}_{2}$ 含量与 BRs 含量和 T-AOC 变化趋势相反。施用外源 BRs (24-epiCS 或 28-homBL) 可显著提高稻穗中内源 BRs (24-epiCS 和 28-homBL)含量与 T-AOC 水平, 并显著降低稻穗中 $\mathrm{H}_{2} \mathrm{O}_{2}$ 含量和颖花退化率, 施用 BRs 合成抑制剂则效果相反。表明 BRs 可以介导氮肥对水稻颖花退化的调控, 在减数分裂期适宜的稻穗含氮量 $(1.25 \%)$ 可有效提高幼穗中的 BRs 含量, 并通过提高抗氧化能力来抑制颖花退化。

关键词: 油菜素甾醇; 氮素; 水稻; 颖花退化

\section{Response of endogenous brassinosteroids to nitrogen rates and its regulatory effect on spikelet degeneration in rice}

YAO Jia-Yu ${ }^{1,2}$, YU Ji-Xiang ${ }^{1,2}$, WANG Zhi-Qin ${ }^{1,2}$, LIU Li-Jun ${ }^{1,2}$, ZHOU Juan ${ }^{1,2}$, ZHANG Wei-Yang ${ }^{1,2, *}$, and YANG Jian-Chang ${ }^{1,2, *}$

\footnotetext{
${ }^{1}$ Jiangsu Key Laboratory of Crop Genetics and Physiology / Jiangsu Key Laboratory of Crop Cultivation and Physiology / Agricultural College of Yangzhou University, Yangzhou 225009, Jiangsu, China; ${ }^{2}$ Jiangsu Co-Innovation Center for Modern Production Technology of Grain Crops / Yangzhou University, Yangzhou 225009, Jiangsu, China
}

\begin{abstract}
In order to investigate whether and how brassinosteroids (BRs) mediate the effect of nitrogen (N) rates on spikelet degeneration of rice, rice cultivars Yangdao 6 and Yongyong 2640 were grown in pots subjected to three $\mathrm{N}$ rates in the whole growth periods. The contents of $\mathrm{N}$, BRs, hydrogen peroxide $\left(\mathrm{H}_{2} \mathrm{O}_{2}\right)$ and total antioxidant capacity (T-AOC) in young rice panicles at meiosis stage and their relationship with spikelet degeneration rate were observed. The results showed that the decreased spikelet degeneration rate was closely associated with enhanced 24-epicastasterone (24-epiCS) and 28-homobrassinolide (28-homBL) contents in young panicles. When $\mathrm{N}$ content of rice panicle was $1.25 \%$, the BRs (24-epiCS and 28 -homBL) content in young
\end{abstract}

本研究由国家自然科学基金项目(31901445，31771710), 江苏省大学生实践创新训练计划项目(201911117010Z), 国家重点研发计划 (2016YFD0300206-4, 2018YFD0300800, 2017YFD0301206), 中国博士后科学基金资助项目(2018M640528), 江苏高校优势学科建设工 程项目(PAPD)和扬州大学高端人才支持计划项目(2015-1)资助。

This study was supported by the Natural Science Foundation of China (31901445, 31771710), the Training Programs of Innovation and Entrepreneurship for Undergraduates of Jiangsu Province (201911117010Z), the Development Program of China (2016YFD0300206-4, 2018YFD0300800, 2017YFD0301206), the Project funded by China Postdoctoral Science Foundation (2018M640528), the Priority Academic Program Development of Jiangsu Higher Education Institutions (PAPD), and the Top Talent Supporting Program of Yangzhou University (2015-01)

*通信作者(Corresponding authors): 张伟杨, E-mail: wyz@yzu.edu.cn; 杨建昌, E-mail: jcyang@yzu.edu.cn 第一作者联系方式: E-mail: yaojiayuyzu@163.com

Received (收稿日期): 2020-07-14; Accepted (接受日期): 2020-11-13; Published online (网络出版日期): 2020-12-23.

URL: https://kns.cnki.net/kcms/detail/11.1809.S.20201223.1138.008.html 
panicle increased significantly, and the spikelet degeneration rate decreased. The variation trend of T-AOC level was very consistent with BRs, and T-AOC was significantly negatively correlated with spikelet degeneration rate, whereas the variation trend of $\mathrm{H}_{2} \mathrm{O}_{2}$ content was opposite to that of T-AOC and BRs contents in the young panicles. Application of exogenous BRs (24-epiCS or 28-homBL) to young panicles could significantly increase the T-AOC level and contents of endogenous 24-epiCS and 28-homBL, but significantly reduce the $\mathrm{H}_{2} \mathrm{O}_{2}$ content and spikelet degeneration rate, while application of BRs synthesis inhibitor had the opposite effect. In summary, BRs mediated the effects of $\mathrm{N}$ application rates on spikelet degeneration, and elevated BRs contents in young panicles could inhibit spikelet degeneration by elevating antioxidant capacity under a proper panicle $\mathrm{N}$ content $(1.25 \%)$ at meiosis stage in rice.

Keywords: brassinosteroids; nitrogen; rice (Oryza sativa L.); spikelet degeneration

水稻是世界上最重要的粮食作物之一, 全球约 有 $50 \%$ 以上的人口以稻米为主食 ${ }^{[1-2]}$ 。为保障粮食安 全, 我国一直将提高单位面积水稻产量作为稻作技 术的一个重要目标 ${ }^{[3-4]}$ 。每穗颖花数是决定水稻产量 的重要因素之一, 且具有较大的变异性和可调性。 因此, 增加每穗颖花数是提高水稻产量的重要途 径 ${ }^{[5-8]}$ 。然而, 颖花退化现象在水稻生产中普遍存在, 尤其是现代培育的一些大穗型品种的颖花退化率更 为严重, 严重制约水稻产量潜力的发挥 ${ }^{[7]}$ 。因此, 如 何减少颖花退化既是重要的科学问题, 也是高产技 术栽培实践需要经常面对的重要技术问题 ${ }^{[7-8]}$ 。多年 来, 国内外学者从农艺学、生理学和遗传学等方面 对水稻颖花退化现象进行了大量研究 ${ }^{[9-12]}$, 但其机 制仍不清楚。

油菜素甾醇(brassinosteroids, BRs)是继生长素、 赤霉素、细胞分裂素、脱落酸和乙烯之后发现的第 六大类植物激素, 主要由油菜素内酯 (brassinolid, BL)和油菜素甾酮(castasterone, CS)及其衍生物组成, 普遍存在于植物界, 广泛参与调节植物的生长发育 过程 ${ }^{[13-15]}$ 。BRs 应用在农业上, 可以增加作物生长 发育速度及种子活力, 促进水稻等作物分蒒、增加 营养体收获量, 提高坐果率, 促进果实肥大, 提高 结实率, 提高籽粒质量, 增强作物抵抗生物和非生 物胁迫等 ${ }^{[13-15]}$ 。当植物缺乏 BRs 时, 会造成植株矮 小、种子数减少并且大量不育、粒重变小 ${ }^{[15-21]}$ 。水 稻颖花发育状况既受基因(内因)控制, 也受营养因 素等外因调节。在所有营养因素中, 氮素是影响作 物产量的最重要因素之一, 合理的氮肥运筹可以减 少颖花退化 ${ }^{[6,22]}$ 。但关于水稻内源 BRs 是否以及如 何对施氮量作出响应, 进而调控颖花退化? 目前尚 不清楚。

与所有需氧有机体一样, 植物在正常呼吸、光 合作用、固氮和环境胁迫应激反应等代谢过程中均 会产生活性氧物质(reactive oxygen species, ROS), 其过量积累通常会引起氧化胁迫, 从而造成细胞受
损甚至死亡, 如过氧化氢 $\left(\mathrm{H}_{2} \mathrm{O}_{2}\right)$ 过量积累会破坏细 胞膜完整性而导致细胞程序性死亡(programmed cell death, PCD), 该过程和水稻颖花退化和败育密切相 关 ${ }^{[9-10,23]}$ 。此外, 众多的研究表明, BRs 可以激活抗 氧化系统活性进而增强植物的抗逆性 ${ }^{[10,23]}$ 。BRs 是 否以通过调控 ROS 的水平来介导施氮量对水稻颖花 退化的影响? 研究报道甚少。鉴此, 本研究较为系 统地观察了在不同施氮量条件下水稻减数分裂期幼 穗中内源 BRs 含量和 ROS 的变化特点及其与颖花退 化的关系, 旨在阐明水稻内源 BRs 对施氮量的响应 特点及其对颖花退化的调控作用。

\section{1 材料与方法}

\section{1 材料和栽培概况}

试验于 2015 年和 2016 年水稻生长季 (5 月至 10 月)在扬州大学江苏省作物栽培生理重点实验室实 验农场开展。供试品种为当地生产中广泛应用且颖 花退化率不同的 2 个水稻品种: 常规籼稻品种扬稻 6 号 (YD-6) 和籼- 粳杂交超级稻品种角优 2640 (YY-2640)。在当地生产中, YD-6 的每穗颖花数通常 在 160 190 之间, YY-2640 的每穗颖花数通常在 250 以上(全生育期施纯氮 $240 \sim 300 \mathrm{~kg} \mathrm{hm}^{-2}$ )。 5 月 11 日至 12 日大田播种育秧, $30 \mathrm{~d}$ 积龄移栽于盆钵 (高 $30 \mathrm{~cm}$, 直径 $25 \mathrm{~cm}$, 容积 $14.72 \mathrm{~L}$ ), 内装过篮大 田表层沙壤土, 含有机质 $2.02 \%$, 碱解氮 $101.5 \mathrm{mg} \mathrm{kg}^{-1}$, 速效磷含量为 $34.2 \mathrm{mg} \mathrm{kg}^{-1}$, 速效钾 含量为 $68.1 \mathrm{mg} \mathrm{kg}^{-1}$ 。田间管理同当地高产栽培技术 进行。各品种在 8 月 10 日至 15 日开始抽穗, 于 10 月 5 日至 6 日收获。

\section{2 实验设计}

每个品种分别设置 3 个施氮水平: 每盆施尿素 低氮 $2 \mathrm{~g}$ (low amount of nitrogen application, LN)、中 氮 $4 \mathrm{~g}$ (moderate amount of nitrogen application, $\mathrm{MN}$ ) 和高氮 $6 \mathrm{~g}$ (high amount of nitrogen application, $\mathrm{HN}$ ), 按照基肥(移栽前 $1 \mathrm{~d}$ ) 40\%: 分菜肥(移栽后 7 d) 20\%: 
促花肥(穗分化始期) 20\%：保花肥(颖花分化期) $20 \%$ 施用。播种前一次性施过磷酸钙(含 $\mathrm{P}_{2} \mathrm{O}_{5} 13.5 \%$ ) 折合纯磷 $0.42 \mathrm{~g}$ 每盆和氯化钾(含 $\mathrm{K}_{2} \mathrm{O} 52 \%$ ) 折合纯 钾 $0.42 \mathrm{~g}$ 每盆。每个处理 80 盆。根据剑叶与倒二叶 的叶耳间距和幼穗镜检相结合的方法来判断幼穗的 分化阶段 ${ }^{[24-25]}$ 。

\section{3 取样与测定}

在分藍早期每个处理选择长势一致的主茎 400 个, 挂上纸牌。于花粉母细胞减数分裂盛期(剑叶与 倒二叶的叶耳间距为零时 ${ }^{[24-25]}$ ) (颖花退化主要发生 在该时期 $\left.{ }^{[6,10]}\right)$, 取各处理 50 个长势一致的, 挂牌主 茎幼穗并逐一检查穗分化阶段，用于测定幼穗中氮 含量、BRs 含量、 $\mathrm{H}_{2} \mathrm{O}_{2}$ 含量和总抗氧化能力(T-AOC) 水平。当 $90 \%$ 的穗抽出时, 各处理取 100 个, 挂牌主 茎穗考查每穗颖花现存数和退化数(白化萎缩的颖 花为退化颖花)。颖花分化数为其现存数和退化数的 总和。

颖花退化率 $(\%)=$ 颖花退化数/颖花分化数 $\times 100$

成熟期收获完整的 10 盆, 考查每盆穗数、每穗 颖花数、结实率和千粒重; 另外, 收获完整的 10 盆 用于考查实产。

\section{4 稻穗含氮量测定}

将在花粉母细胞减数分裂期盛期所取的稻穗烘 干后粉碎过篮，用 FOSS 公司的 Kjeltec 8400 全自动 凯氏定氮仪测定稻穗含氮量。

\section{5 油菜素甾醇(BRs)的提取与测定}

稻穗中内源 BRs 的提取与纯化参照 Ding 等 ${ }^{[26]}$ 的方法, 略作改进：4 6 g 鲜样组织用液氮冷冻, 用 研钵磨成细粉, $0.5 \sim 0.8 \mathrm{~g}$ 粉末转移到一个 $10 \mathrm{~mL}$ 离 心管内, 加乙腈 $\left(5 \mathrm{~mL} \mathrm{~g}^{-1}\right)$ 提取, $-20^{\circ} \mathrm{C}$ 冰箱中保存一 夜待用。乙腈提取的样品在 $4^{\circ} \mathrm{C}, 10,000 \mathrm{r} \mathrm{min}^{-1}$ 下离 心 $10 \mathrm{~min}$ 。收集上清液, 剩余残渣用 $1 \mathrm{~mL}$ 乙腈再次 提取, 合并两部分上清液, 在温和的氮气流中蒸干 浓缩备用。根据 Chen 等 ${ }^{[27]}$ 报道的方法, 进行萃取、 脱水和双层固相萃取(DL/SPE), 收集萃取液并在温 和的氮气流中蒸干浓缩。采用液质联用仪 (LC-MS/MS, TSQ Vantage, Thermo Fisher Scientific, Waltham, MA，USA)的多级反应模式(MRM)下进行 测定与分析。前人报道, 24- 表油菜素甾酮 (24-epicastasterone，24-epiCS) 和 28-高油菜素内酯 (28-homobrassinolide, 28-homoBL) 是水稻体内高生物活 性且重要的 $\mathrm{BRs}^{[6,10,28]}$, 因此本研究主要测定 24-epiCS 和 28-homoBL, 以 $\mathrm{pmol} \mathrm{g}^{-1} \mathrm{DW}$ (干重)表示其含量。
1.6 总抗氧化能力 $(T-A O C)$ 和过氧化氢 $\left(\mathrm{H}_{2} \mathrm{O}_{2}\right)$ 含 量的测定

稻穗中 $\mathrm{T}-\mathrm{AOC}$ 水平的测定, 参照 $\mathrm{T}-\mathrm{AOC}$ 试剂盒 生产商(苏州科铭生物技术有限公司)制定的方法进 行测定与分析, 单位以 $\mathrm{U} \mathrm{g}^{-1} \mathrm{DW}$ 表示。 $\mathrm{H}_{2} \mathrm{O}_{2}$ 含量测 定采用 Rao 等 ${ }^{[29]}$ 报道的方法, 单位以 $\mu \mathrm{mol} \mathrm{g} \mathrm{g}^{-1} \mathrm{DW}$ 表示。

\section{7 化学调控物质处理}

供试品种为扬稻 6 号和角优 2640, 栽培方法与 生长条件同 MN 处理(2016 年), 120 盆重复。在分薛 早期将所有主茎挂上纸牌, 在花粉母细胞减数分裂 前期(剑叶的叶耳低于倒二叶的叶耳 $5 \mathrm{~cm}$ 时)用 $1 \mathrm{~mL}$ 注射器(针孔直径 $0.33 \mathrm{~mm}$, 美国 $\mathrm{BD}$ 公司生产)往长 势一致的主茎幼穗中分别注射 $10 \mathrm{nmol} \mathrm{L}{ }^{-1} 24$-epiCS (T1), $10 \mathrm{nmol} \mathrm{L}^{-1}$ 28-homoBL (T2), $10 \mathrm{nmol} \mathrm{L}{ }^{-1}$ brassinazole (BRZ，芸薹素唑, BRs 合成抑制剂) (T3), $10 \mathrm{nmol} \mathrm{L}{ }^{-1} 24$-epiCS + $10 \mathrm{nmol} \mathrm{L}{ }^{-1} \mathrm{BRZ}$ (T4)。喷施 的所有溶液中均含有 $0.1 \%(\mathrm{v} / \mathrm{v})$ 的乙醇和 $0.01 \%(\mathrm{v} / \mathrm{v})$ Tween 20 作为展开剂, 用含有 $0.1 \%(\mathrm{v} / \mathrm{v})$ 的乙醇和 $0.01 \%(\mathrm{v} / \mathrm{v})$ Tween 20 的清水作为对照 $(\mathrm{CK})$ 。每茎注 射 $0.5 \mathrm{~mL}$, 每处理 120 茎。为保证注射效果, 连续 处理 $4 \mathrm{~d}$, 注射操作对幼穗未见明显伤害。在花粉母 细胞减数分裂盛期, 每处理取 40 个幼穗并逐一检查 穗分化阶段, 用于测定 BRs 含量、 T-AOC 水平和 $\mathrm{H}_{2} \mathrm{O}_{2}$ 含量; 抽穗期取 40 个稻穗考察颖花分化与退 化状况; 成熟期取 40 个稻穗考察单穗产量构成因 素, 测定方法同上述。

\section{8 数据处理与计算方法}

数据用 Microsoft Excel 2010、SPSS 16.0 和 SAS (version 9.2, SAS Institute), 进行统计分析, 用 $P=0.05$ 最小显著极差法 $\left(\mathrm{LSD}_{0.05}\right)$ 进行平均数显著性检验。

\section{2 结果与分析}

\section{1 颖花发育与产量}

施氮量对颖花发育与产量的影响因品种而异。 随着施氮量的增加, 甬优 2640 的颖花分化数和每穗 颖花数显著增加, 颖花退化率显著降低。扬稻 6 号 在 LN 处理下颖花分化数和每穗颖花数最少, 在 MN 处理下最多; 颖花退化率在 MN 处理下最低, 在 $\mathrm{HN}$ 处理下最高(表 1)。两品种的产量在处理间的变化均 表现为: $M N$ 处理的最高, $\mathrm{LN}$ 处理的最低; HN 处理 的产量低于 $\mathrm{MN}$ 处理的重要原因是结实率和千粒重 的降低。2 年结果趋势相同(表 1)。 
表 1 不同施氮量处理对水稻颖花发育、产量和产量构成因素的影响

Table 1 Effects of different nitrogen rates on spikelet development, grain yield and yield components in rice

\begin{tabular}{|c|c|c|c|c|c|c|c|}
\hline $\begin{array}{c}\text { 年份/品种/处理 } \\
\text { Year/cultivar/treatment }\end{array}$ & $\begin{array}{c}\text { 每盆穗数 } \\
\text { Number of } \\
\text { panicle } \\
\end{array}$ & $\begin{array}{c}\text { 每穗分化颖花数 } \\
\text { Differentiated } \\
\text { spikelets per panicle }\end{array}$ & $\begin{array}{c}\text { 颖花退化率 } \\
\text { Spikelet degen- } \\
\text { eration rate (\%) }\end{array}$ & $\begin{array}{l}\text { 每穗粒数 } \\
\text { Spikelets per } \\
\text { panicle }\end{array}$ & $\begin{array}{c}\text { 结实率 } \\
\text { Fully-filled } \\
\text { grains rate }(\%)\end{array}$ & $\begin{array}{c}\text { 千粒重 } \\
\text { 1000-grain } \\
\text { weight }(\mathrm{g}) \\
\end{array}$ & $\begin{array}{c}\text { 产量 } \\
\text { Grain yield } \\
\left(\mathrm{g} \mathrm{pot}^{-1}\right) \\
\end{array}$ \\
\hline \multicolumn{8}{|l|}{2015} \\
\hline \multicolumn{8}{|l|}{ 扬稻 6 号 YD-6 } \\
\hline 低氮 LN & $15.0 \pm 0.56 \mathrm{c}$ & $188 \pm 2.69 \mathrm{c}$ & $8.32 \pm 0.29 \mathrm{~b}$ & $170 \pm 2.95 \mathrm{~b}$ & $89.1 \pm 0.98 \mathrm{a}$ & $28.3 \pm 0.48 \mathrm{a}$ & $62.9 \pm 2.20 \mathrm{c}$ \\
\hline 中氮 MN & $19.9 \pm 0.72 b$ & $201 \pm 2.87 \mathrm{a}$ & $7.49 \pm 0.04 \mathrm{c}$ & $181 \pm 4.22 \mathrm{a}$ & $86.5 \pm 1.03 \mathrm{~b}$ & $27.0 \pm 0.53 \mathrm{ab}$ & $83.7 \pm 2.55 \mathrm{a}$ \\
\hline 高氮 HN & $22.3 \pm 0.91 \mathrm{a}$ & $195 \pm 2.78 b$ & $9.68 \pm 0.12 \mathrm{a}$ & $172 \pm 2.21 \mathrm{~b}$ & $80.0 \pm 0.71 \mathrm{c}$ & $25.9 \pm 0.83 \mathrm{~b}$ & $78.2 \pm 2.91 \mathrm{~b}$ \\
\hline \multicolumn{8}{|l|}{ 角优 2640 YY-2640 } \\
\hline 低氮 LN & $14.2 \pm 0.57 \mathrm{~b}$ & $293 \pm 5.79 \mathrm{c}$ & $14.9 \pm 0.16 \mathrm{a}$ & $245 \pm 5.06 \mathrm{~b}$ & $84.4 \pm 1.80 \mathrm{a}$ & $25.4 \pm 0.37 \mathrm{a}$ & $73.6 \pm 3.47 \mathrm{~b}$ \\
\hline 中氮 $\mathrm{MN}$ & $17.8 \pm 0.23 \mathrm{a}$ & $336 \pm 4.80 \mathrm{~b}$ & $13.4 \pm 0.34 \mathrm{~b}$ & $276 \pm 3.73 \mathrm{a}$ & $78.7 \pm 2.66 \mathrm{~b}$ & $24.8 \pm 0.38 \mathrm{a}$ & $95.3 \pm 1.61 \mathrm{a}$ \\
\hline 高氮 HN & $18.5 \pm 0.40 \mathrm{a}$ & $347 \pm 4.96 \mathrm{a}$ & $11.8 \pm 0.27 \mathrm{c}$ & $282 \pm 5.76 \mathrm{a}$ & $72.7 \pm 2.07 \mathrm{c}$ & $24.5 \pm 0.27 \mathrm{a}$ & $91.6 \pm 3.41 \mathrm{a}$ \\
\hline \multicolumn{8}{|l|}{2016} \\
\hline \multicolumn{8}{|l|}{ 扬稻 6 号 YD-6 } \\
\hline 低氮 LN & $14.4 \pm 0.36 \mathrm{c}$ & $185 \pm 2.45 \mathrm{~b}$ & $8.52 \pm 0.56 \mathrm{ab}$ & $172 \pm 2.25 \mathrm{~b}$ & $90.3 \pm 1.59 \mathrm{a}$ & $28.6 \pm 0.70 \mathrm{a}$ & $65.5 \pm 2.38 \mathrm{c}$ \\
\hline 中氮 $\mathrm{MN}$ & $19.5 \pm 0.83 b$ & $198 \pm 3.19 \mathrm{a}$ & $7.55 \pm 0.31 \mathrm{~b}$ & $179 \pm 3.43 \mathrm{a}$ & $87.1 \pm 0.99 \mathrm{~b}$ & $27.3 \pm 0.75 \mathrm{ab}$ & $85.0 \pm 2.70 \mathrm{a}$ \\
\hline 高氮 HN & $22.1 \pm 0.76 \mathrm{a}$ & $191 \pm 3.01 \mathrm{ab}$ & $9.36 \pm 0.47 \mathrm{a}$ & $174 \pm 2.06 \mathrm{ab}$ & $79.0 \pm 1.72 \mathrm{c}$ & $26.1 \pm 1.14 \mathrm{~b}$ & $80.2 \pm 4.42 b$ \\
\hline \multicolumn{8}{|l|}{ 角优 2640 YY-2640 } \\
\hline 低氮 LN & $13.8 \pm 0.75 \mathrm{c}$ & $292 \pm 5.76 b$ & $15.8 \pm 0.33 \mathrm{a}$ & $241 \pm 7.12 b$ & $84.1 \pm 2.33 \mathrm{a}$ & $25.6 \pm 0.52 \mathrm{a}$ & $73.1 \pm 4.05 b$ \\
\hline 中氮 $\mathrm{MN}$ & $17.4 \pm 0.41 \mathrm{~b}$ & $330 \pm 6.23 \mathrm{a}$ & $13.8 \pm 0.32 \mathrm{~b}$ & $272 \pm 5.69 \mathrm{a}$ & $80.7 \pm 1.22 \mathrm{~b}$ & $25.0 \pm 0.60 \mathrm{ab}$ & $97.3 \pm 3.01 \mathrm{a}$ \\
\hline 高氮 HN & $18.9 \pm 0.61 \mathrm{a}$ & $341 \pm 4.59 \mathrm{a}$ & $12.5 \pm 0.29 \mathrm{c}$ & $278 \pm 8.58 \mathrm{a}$ & $74.3 \pm 1.53 \mathrm{c}$ & $24.1 \pm 0.49 \mathrm{~b}$ & $93.6 \pm 4.58 \mathrm{a}$ \\
\hline
\end{tabular}

数据为平均值 \pm 标准误, 同一年份相同品种标以不同小写字母的值在处理间 0.05 水平上差异显著 $(n=10)$ 。

LN, MN, and HN denote low amount, moderate amount and high amount of nitrogen application, respectively. YD-6: Yangdao 6; YY-2640: Yongyou 2640. Data are mean \pm SE with ten independent replications; values followed by different letters indicate significant differences at the 0.05 probability level within the same column at the same year and cultivar $(n=10)$.

\section{2 稻穗含氮量和 BRs 含量}

2 个品种减数分裂期稻穗含氮量均随着施氮量 的增加明显增加(图 1-A, B)。减数分裂期稻穗中 BRs (24-epiCS 和 28-homoBL)的含量在处理间的变化因 施氮量和品种的不同而异。甬优 2640 幼穗中

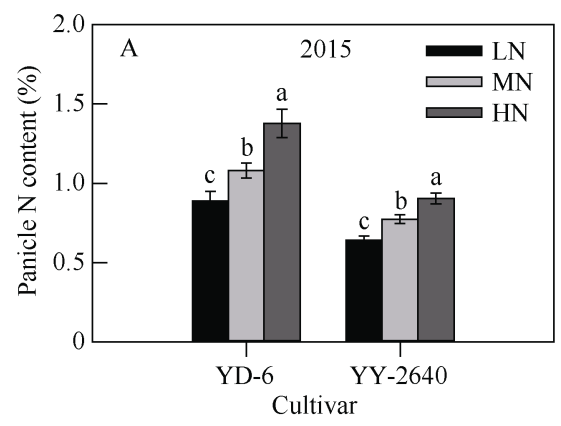

24-epiCS 和 28 -homoBL 的含量随施氮量的提高而显 著增加。扬稻 6 号幼穗中的 24-epiCS 和 28-homoBL 含 量均在 HN 处理下最低, 在 MN 处理下最高(图 2-A D)。 相同氮处理下, 扬稻 6 号的稻穗含氮量和 BRs 含量 均明显高于角优 2640 (图 1-A, B 和图 2-A D)。

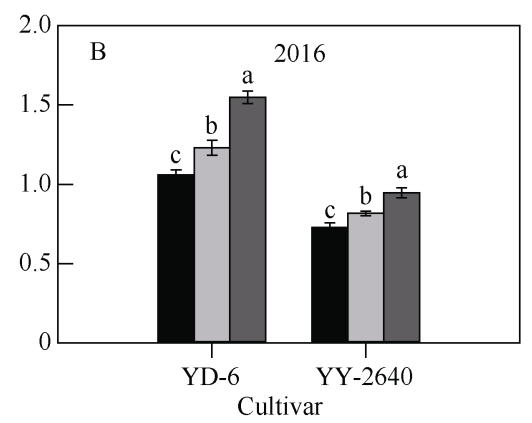

图 1 不同施氮量处理对稻穗含氮量 $(A, B)$ 的影响

Fig. 1 Effects of different nitrogen rates on nitrogen content $(A, B)$ of young panicles in rice

缩略词同表 1。数据为平均值土标准误, 品种柱上方不同字母表示 0.05 水平上差异显著(同一品种内比较, $n=5$ )

Abbreviations are the same as those given in Table 1. Vertical bars represent mean \pm SE $(n=5)$. Different letters above bars indicate significant differences at the 0.05 probability level within the same cultivar. 

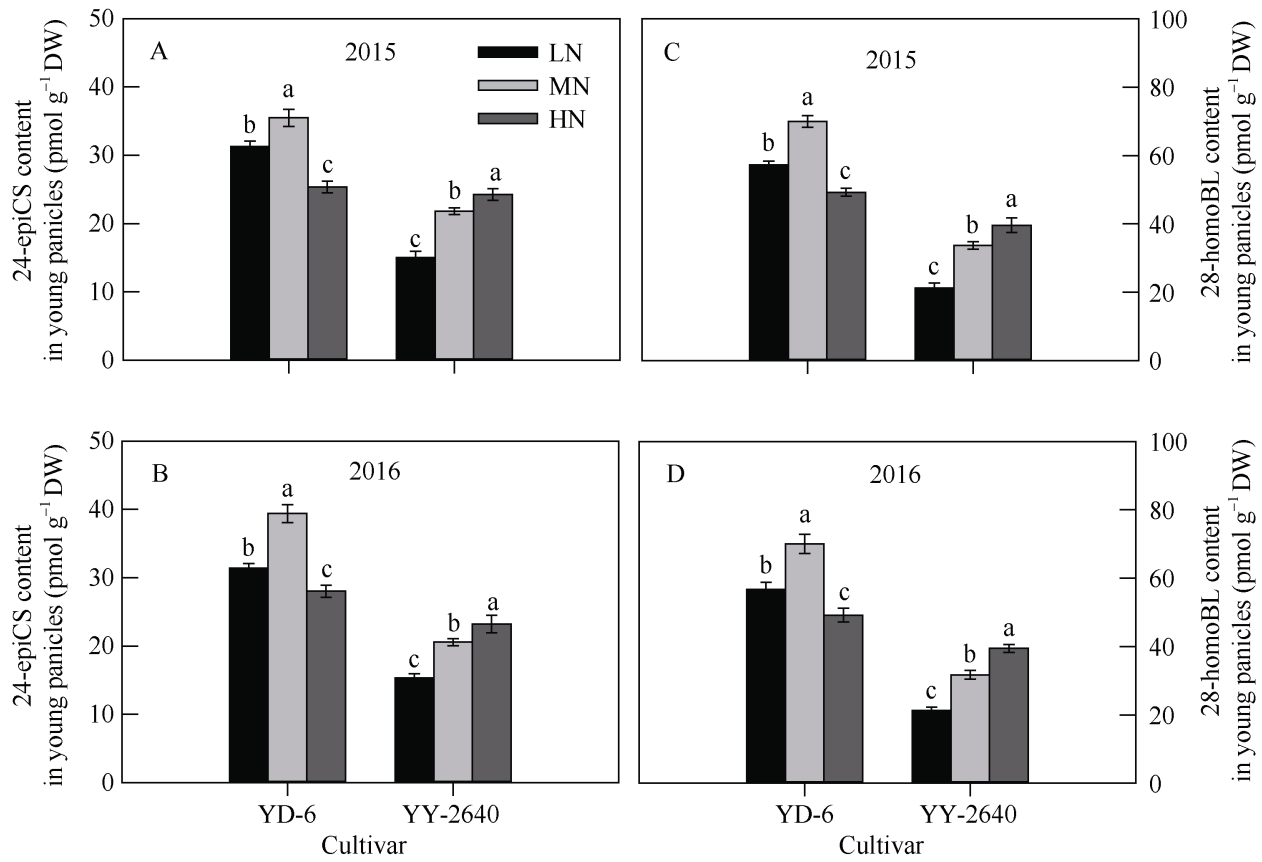

图 2 不同施氮量处理对稻穗中 24-表油菜素甾酮(24-epiCS) (A, B)和 28-高油菜素内酯(28-homoBL) (C, D)含量的影响

Fig. 2 Effects of different nitrogen rates on the contents of 24-epicastasterone (24-epiCS) (A, B) and 28-homobrassinolide (28-homoBL) (C, D) of young panicles in rice

缩写同表 1 。数据为平均值 \pm 标准误, 品种柱上方不同字母表示 0.05 水平上差异显著(同一品种内比较, $n=3$ )。

Abbreviations are the same as those given in Table 1. Vertical bars represent \pm SE of the mean $(n=3)$. Different letters above bars indicate significant differences at the 0.05 probability level within the same cultivar.

\section{3 稻穗中 T-AOC 水平和 $\mathrm{H}_{2} \mathrm{O}_{2}$ 含量}

减数分裂期稻穗中 $\mathrm{T}-\mathrm{AOC}$ 水平在处理间的变化

与 BRs (24-epiCS 和 28-homoBL)的含量变化一致。 扬稻 6 号幼穗中的 T-AOC 水平在 HN 处理下最低, 在 MN 处理下最高, 角优 2640 幼穗中的 T-AOC 水 平随施氮量的增加而显著提高(图 3-A, B)。与之相反 的是，扬稻 6 号幼穗中的 $\mathrm{H}_{2} \mathrm{O}_{2}$ 含量在 $\mathrm{MN}$ 处理下最 低, HN 处理下最高(图 3-C, D), 而甬优 2640 幼穗中 的 $\mathrm{H}_{2} \mathrm{O}_{2}$ 含量随施氮量的增加而显著降低(图 3-C, D)。 相同氮处理下，扬稻 6 号的稻穗中 T-AOC 水平显 著高于角优 2640 稻穗中的含量, 而扬稻 6 号的稻 穗中 $\mathrm{H}_{2} \mathrm{O}_{2}$ 含量显著低于角优 2640 稻穗中的含量 (图 3-A D)。

\section{4 相关分析}

减数分裂期稻穗中 BRs (24-epiCS 和 28-homoBL)含量和 T-AOC 水平均与稻穗含氮量呈 开口向下的二次曲线函数关系: $C=$ $-59.5+152 x-62.1 x^{2}, \mathrm{~B}=-146+338 x-137 x^{2}$ 和 $T=$ $-137+332 x-138 x^{2}$ ( $C$ 代表 24-epiCS 含量, $B$ 代表 28-homoBL 含量, $T$ 代表 T-AOC, $x$ 代表稻穗含氮量; $\left.R^{2}=0.666^{*} \sim 0.758^{* *}\right)$ (图 4-A C )。稻穗中 $\mathrm{H}_{2} \mathrm{O}_{2}$ 含量
和颖花退化率均与稻穗含氮量呈开口向上的二次曲 线函数 $H=59.7-83.9 x+34.3 x^{2}$ 和 $S=41.5-52.4 x+$ $20.6 x^{2}$ ( $H$ 代表 $\mathrm{H}_{2} \mathrm{O}_{2}$ 含量, $S$ 代表颖花退化率; $R^{2}=$ $0.746^{* *} \sim 0.831^{* *}$ ) (图 4-D, E)。此外, 稻穗中 T-AOC 水平与 BRs (24-epiCS 和 28-homoBL)含量显著正相 关 $\left(r=0.974^{* *} \sim 0.982^{* *}\right)$, 而稻穗中 $\mathrm{H}_{2} \mathrm{O}_{2}$ 含量和颖花 退化率均与 BRs (24-epiCS 和 28-homoBL) 含量显著 负相关 $\left(r=-0.956^{* *} \sim-0.978^{* *}\right)$ (图 5-A $\sim \mathrm{F}$ )。

\section{5 外源化学调控物质对颖花退化的影响}

与对照 $(\mathrm{CK})$ 相比, 在减数分裂前期对稻穗施用 外源 BRs (24-epiCS 或 28-homoBL)处理, 可显著增 加幼穗中内源 BRs (24-epiCS 或 28-homoBL)含量和 $\mathrm{T}-\mathrm{AOC}$ 水平, 显著减少幼穗中 $\mathrm{H}_{2} \mathrm{O}_{2}$ 含量, 使颖花退 化率显著降低, 每穗颖花数和结实率均显著增加。 施用 BRZ (BRs 合成抑制剂)则效果相反, 即幼穗中 内源 BRs (24-epiCS 或 28-homoBL)含量和 T-AOC 水 平显著降低, 幼穗中 $\mathrm{H}_{2} \mathrm{O}_{2}$ 含量大幅增加, 颖花退化 率显著增加, 每穗颖花数和结实率均显著降低。当 BRZ 联合 24-epiCS 一起施用, 则可有效解除 BRZ 对颖花发育与产量形成的抑制作用。外源化学调控 物质处理对粒重影响较小(表 2 和表 3)。 

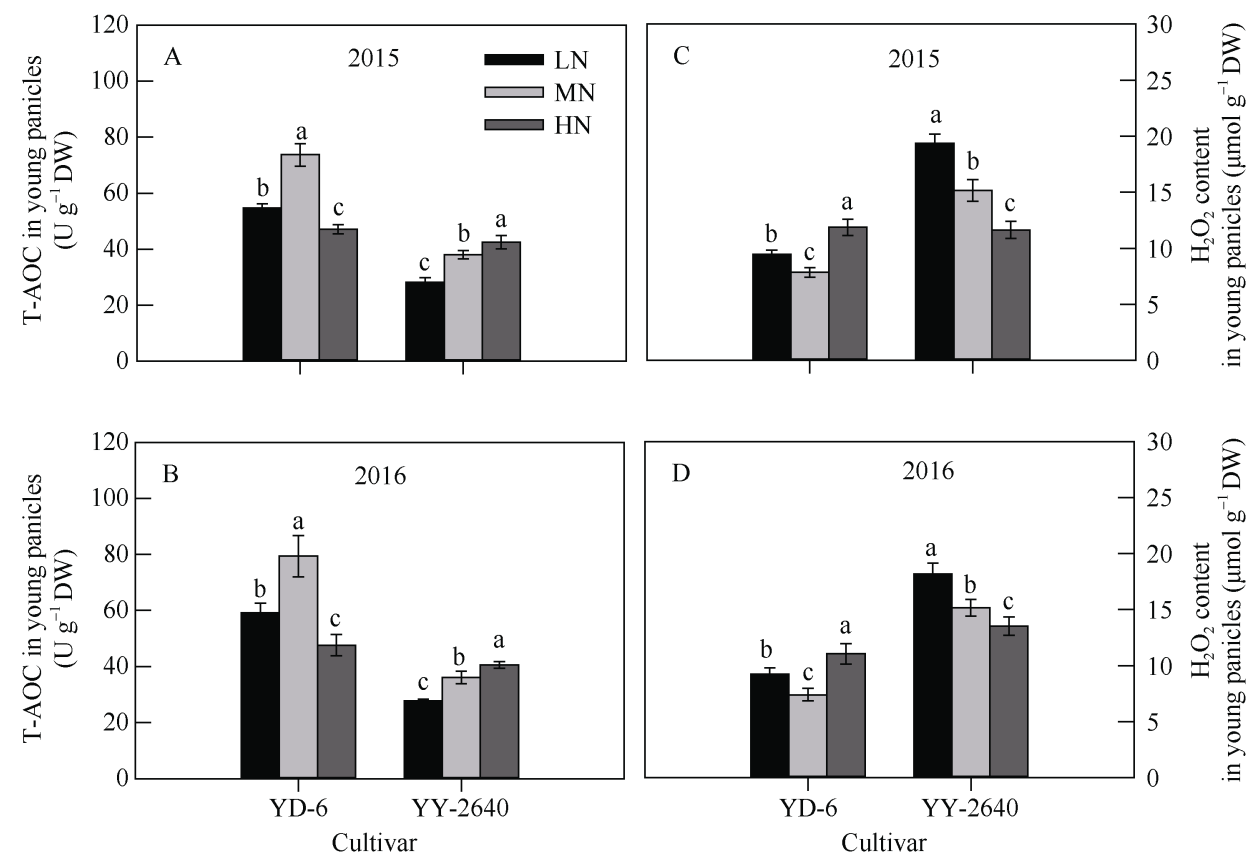

图 3 不同施氮量处理对稻穗中总抗氧化能力 (T-AOC) $(A, B)$ 和过氧化氢 $\left(\mathrm{H}_{2} \mathrm{O}_{2}\right)(\mathrm{C}, \mathrm{D})$ 含量的影响

Fig. 3 Effects of different nitrogen rates on the total antioxidant capacity (T-AOC) level $(\mathrm{A}, \mathrm{B})$ and hydrogen peroxide $\left(\mathrm{H}_{2} \mathrm{O}_{2}\right)$ content $(C, D)$ of young panicles in rice

缩写同表 1 。数据为平均值 \pm 标准误, 品种柱上方不同字母表示在 0.05 水平上差异显著(同一品种内比较, $n=3$ )。

Abbreviations are the same as those given in Table 1. Vertical bars represent mean \pm SE $(n=3)$. Different letters above bars indicate significant differences at the 0.05 probability level within the same cultivar.

\section{3 讨论}

通常认为, 水稻每穗粒数与穗发育期植株含氮 量呈线性正相关; 在水稻穗发育期施用氮肥能够显 著影响颖花发育, 有效增加每穗颖花数 ${ }^{[22,31-32]}$ 。因此, 在水稻生产中通过施用氮素穗肥, 以增加每穗颖花 数进而提高产量 ${ }^{[33-34]}$ 。本研究观察到, 施氮量对降 低颖花退化率的作用因水稻品种的类型差异而不 同。对于每穗颖花分化数较少的品种扬稻 6 号而言, 与 $\mathrm{LN}$ 或 MN 处理相比, HN 处理下会显著增加颖花 退化率, 而对于每穗颖花分化数较多的品种甬优 2640 而言, 与 $\mathrm{LN}$ 或 MN 处理相比, HN 处理下能有 效降低颖花退化率(表 1)。这些结果表明, 颖花分化 数较多的水稻品种可能比每穗颖花数较少的品种需 要更高的氮素供应, 以减少颖花退化, 但施氮量过 高, 特别是对颖花分化数较少的品种, 可能会增加 水稻颖花退化。

不同穗型的水稻品种的颖花退化率对施氮量响 应差异的机制尚不清楚。已有研究表明, BRs 在增加 水稻颖花数量方面起着重要作用 ${ }^{[6,10,35]}$ 。本研究观察 到, 不同施氮量可以调控减数分裂期稻穗中 BRs (24-epiCS 和 28-homoBL) 含量, 且 BRs (24-epiCS 和
28-homoBL) 含量与颖花退化率显著负相关 (图 2-A D 和图 5-E, F)。当外源 BRs (24-epiCS 或 28-homoBL)施用于减数分裂期时期的稻穗, 可显著 增加稻穗内源 BRs (24-epiCS 和 28-homoBL)含量、 有效减少颖花退化率, 显著提高每穗粒数和产量。 当施用 BRZ (BRs 合成抑制剂)则效果相反, 且施用 外源 24-epiCS 可有效解除 BRZ 对颖花发育与产量 形成的抑制作用(表 2 和表 3 )。这些结果表明, 在水 稻幼穗发育期合理施用氮肥可有效提高稻穗中 BRs 含量, 进而显著减少水稻颖花退化。

本研究观察到颖花退化率和稻穗中 BRs (24-epiCS 和 28-homoBL)含量显著负相关, 且它们 均与稻穗含氮量呈二次多项式关系, 说明减数分裂 期穗中 BRs 含量越高, 颖花退化率越低; 当减数分 裂期稻穗的含氮量为 $1.25 \%$ 时, BRs 含量最高, 颖花 退化率最低(图 4-A, B, E; 图 5-E, F)。这在一定程度 上解释了为什么与 $\mathrm{LN}$ 或 MN 相比, HN 处理会使每 穗颖花数较少的品种扬稻 6 号的颖花退化率明显增 加, 主要因为幼穗中过高的氮含量可能会降低 BRs (24-epiCS 或 28-homoBL) 含量; 但大穗型水稻品种 角优 2640 相对较大的生物量会对植株的氮素含量 起稀释作用, 因而在 $\mathrm{HN}$ 条件下稻穗中的氮含量仍 

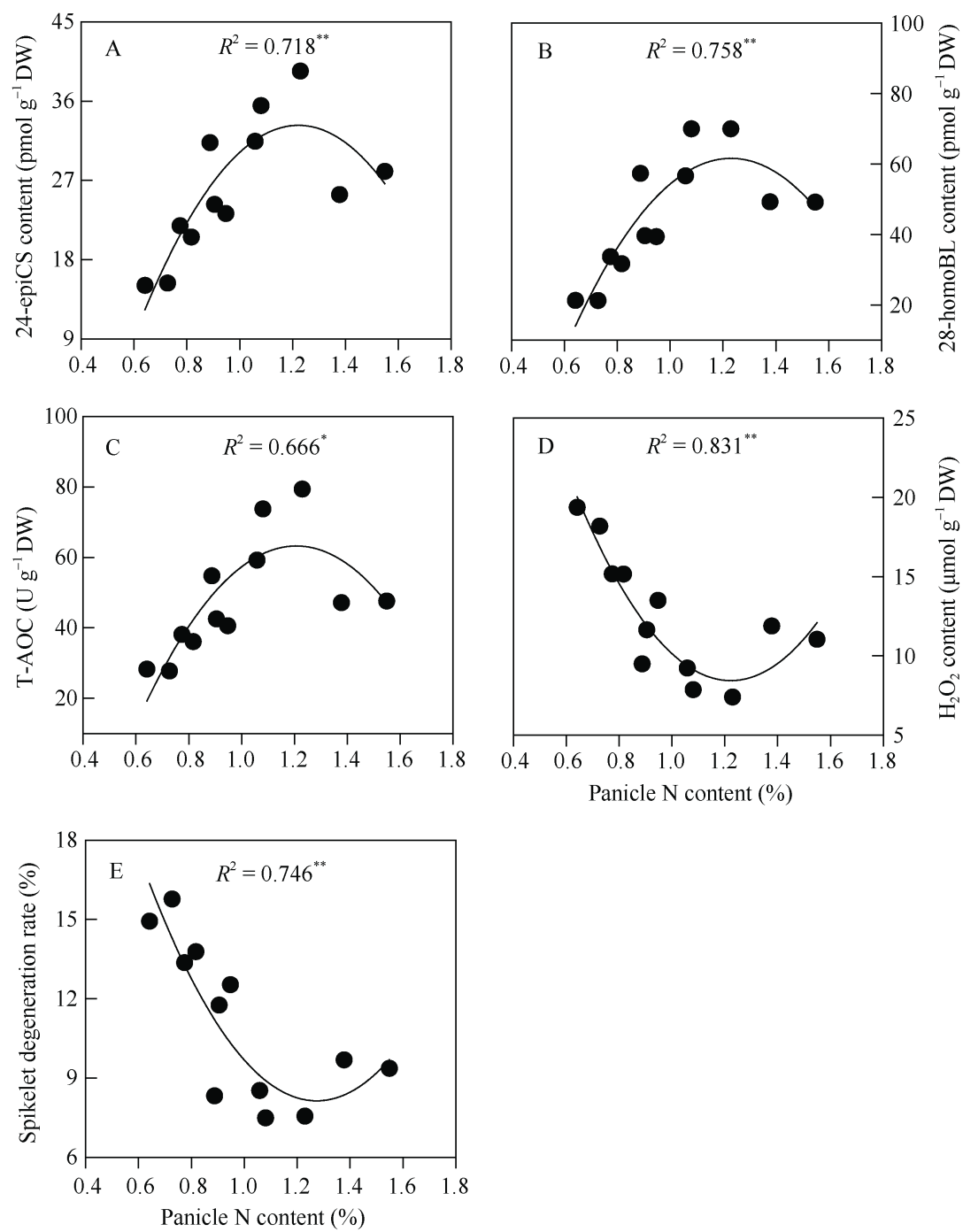

图 4 稻穗含氮量与油菜素甾醇 $(B R s)(A, B)$ 、总抗氧化能力 $(T-A O C)(C)$ 、过氧化氢 $\left(H_{2} \mathrm{O}_{2}\right)(D)$ 含量和颖花退化率 $(E)$ 的关系

Fig. 4 Correlations of panicle nitrogen content with brassinosteroids (BRs) contents (A, B), total antioxidant capacity (T-AOC) level (C), and $\mathrm{H}_{2} \mathrm{O}_{2}$ content (D), and spikelet degeneration rate (E) of young panicles in rice

${ }^{*},{ }^{* *}$ 分别代表在 0.05 和 0.01 水平上差异显著 $(n=12)$ 。

${ }^{*}$ and ${ }^{* *}$ represent significant differences at the 0.05 and 0.01 probability levels, respectively $(n=12)$.

然相对适宜, 进而增加稻穗 BRs 含量, 减少颖花退 化。这些结果表明，水稻减数分裂期 $1.25 \%$ 的稻穗含 氮量可作为氮肥施用的一个重要标准, 可有效提高 幼穗 BRs 水平, 减少颖花退化, 进而提高水稻库容 与产量潜力。

关于 BRs 调控水稻颖花退化的机制，以往的 相关研究甚少。前人报道, 发育稻穗中产生或积 累过多的 $\mathrm{H}_{2} \mathrm{O}_{2}$ 可以通过激发水稻幼穗中细胞凋 亡相关基因的表达来诱导 PCD, 进而导致颖花退 化 ${ }^{[10,23,36-37]}$ 。本研究观察到, 稻穗中的 $\mathrm{H}_{2} \mathrm{O}_{2}$ 含量与 BRs (24-epiCS 和 28-homoBL)含量显著负相关, 与 颖花退化率显著正相关，而幼穗中 T-AOC 水平与 $\mathrm{H}_{2} \mathrm{O}_{2}$ 含量变化趋势相反(图 5-A F)。对减数分裂期
的稻穗外源施用 BRs (24-epiCS 或 28-homoBL) 可显 著提高稻穗中 T-AOC 水平, 降低 $\mathrm{H}_{2} \mathrm{O}_{2}$ 含量, 进而降 低颖花退化率, 增加每穗颖花数, 而施用 BRs 合成 抑制剂的效果则相反(表 2 和表 3)。这些结果表明, 适量施用氮肥或使减数分裂期稻穗含氮量为 $1.25 \%$ 时, 可以有效提高稻穗中 BRs 含量, 增强抗氧化能 力, 减轻减数分裂期间 ROS 的对颖花细胞的伤害, 保护分化的颖花免于退化。

值得注意的是, 在本研究中观察到颖花退化率 在品种间的差异大于氮肥处理间的差异(扬稻 6 号和 甬优 2640 在氮肥处理间的颖花退化率分别介于 7.49\% 9.68\%和 11.8\% 15.8\%)（表 1)，这一方面表明 水稻颖花退化状况在很大程度上受内在遗传力 $($ 内 

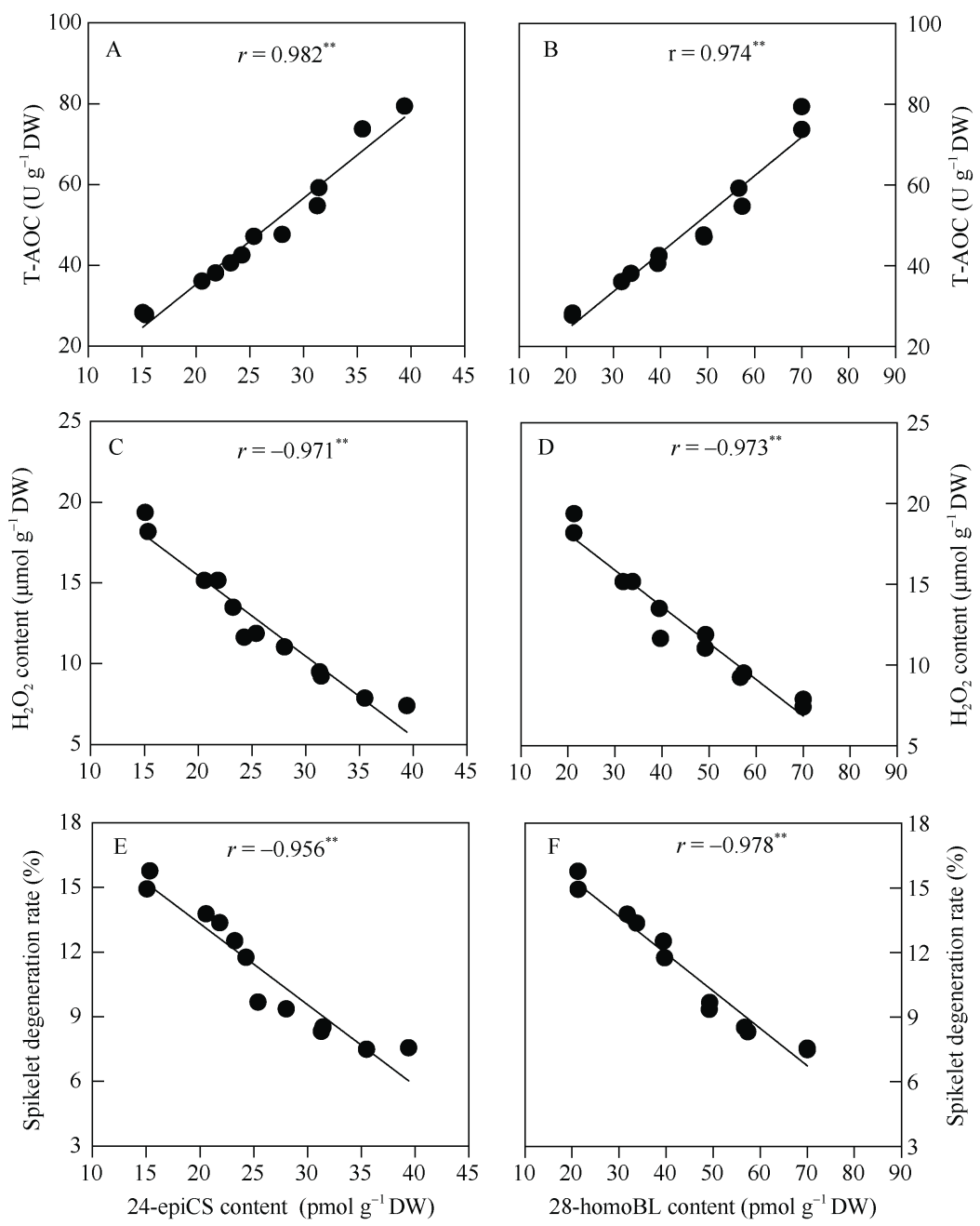

图 5 稻穗中油菜素甾醇 $(B R s)$ 含量与总抗氧化能力 $(T-A O C)(A, B)$ 、过氧化氢 $\left(H_{2} \mathrm{O}_{2}\right)$ 含量 $(C, D)$ 和颖花退化率 $(E, F)$ 的关系

Fig. 5 Correlations of brassinosteroids (BRs) contents with total antioxidant capacity (T-AOC) $(A, B)$, hydrogen peroxide $\left(\mathrm{H}_{2} \mathrm{O}_{2}\right)$ content $(C, D)$, and spikelet degeneration rate $(E, F)$ of young panicles in rice

**表示在 0.05 和 0.01 水平上差异显著 $(n=12)$ 。

${ }^{*}$ and ${ }^{* *}$ represent significant differences at the 0.05 and 0.01 probability levels, respectively $(n=12)$.

表 2 外源化学调控物质对稻穗中油菜素甾醇(BRs)、总抗氧化能力(T-AOC)和过氧化氢 $\left(\mathrm{H}_{2} \mathrm{O}_{2}\right)$ 水平的影响

Table 2 Effects of chemical applications on the levels of brassinosteroids (BRs), hydrogen peroxide $\left(\mathrm{H}_{2} \mathrm{O}_{2}\right)$, and total antioxidant capacity (T-AOC) of young panicles in rice

\begin{tabular}{|c|c|c|c|c|c|c|}
\hline \multirow{2}{*}{$\begin{array}{c}\text { 品种 } \\
\text { Cultivar }\end{array}$} & \multirow{2}{*}{$\begin{array}{c}\text { 生理指标 } \\
\text { Physiological parameter }\end{array}$} & \multicolumn{5}{|c|}{ 化学物质处理 Chemical treatment } \\
\hline & & $\mathrm{CK}$ & $\mathrm{T} 1$ & $\mathrm{~T} 2$ & $\mathrm{~T} 3$ & $\mathrm{~T} 4$ \\
\hline 扬稻 6 号 & 24-epiCS (pmol g $\left.{ }^{-1} \mathrm{DW}\right)$ & $37.9 \pm 2.28 \mathrm{c}$ & $58.6 \pm 2.61 \mathrm{a}$ & $50.4 \pm 1.72 b$ & $14.8 \pm 1.01 \mathrm{~d}$ & $37.2 \pm 2.26 \mathrm{c}$ \\
\hline \multirow[t]{3}{*}{ YD-6 } & 28-homoBL (pmol g-1 DW) & $73.7 \pm 3.54 \mathrm{c}$ & $106 \pm 4.99 \mathrm{~b}$ & $126 \pm 3.62 \mathrm{a}$ & $35.4 \pm 2.07 \mathrm{~d}$ & $79.3 \pm 4.12 \mathrm{c}$ \\
\hline & T-AOC $\left(\mathrm{U} \mathrm{g}^{-1} \mathrm{DW}\right)$ & $80.3 \pm 2.58 \mathrm{~b}$ & $114 \pm 4.09 \mathrm{a}$ & $119 \pm 5.08 \mathrm{a}$ & $34.6 \pm 2.46 \mathrm{c}$ & $79.8 \pm 2.26 b$ \\
\hline & $\mathrm{H}_{2} \mathrm{O}_{2}\left(\mu \mathrm{mol} \mathrm{g}{ }^{-1} \mathrm{DW}\right)$ & $7.68 \pm 0.78 b$ & $4.24 \pm 0.26 \mathrm{c}$ & $4.17 \pm 0.25 \mathrm{c}$ & $17.76 \pm 0.56 \mathrm{a}$ & $7.76 \pm 0.68 \mathrm{~b}$ \\
\hline 角优 2640 & 24-epiCS (pmol g $\left.{ }^{-1} \mathrm{DW}\right)$ & $21.5 \pm 1.18 \mathrm{c}$ & $38.5 \pm 1.45 \mathrm{a}$ & $31.3 \pm 1.21 \mathrm{~b}$ & $10.8 \pm 0.48 \mathrm{~d}$ & $21.9 \pm 0.83 \mathrm{c}$ \\
\hline \multirow[t]{3}{*}{ YY-2640 } & 28-homoBL (pmol g $\left.{ }^{-1} \mathrm{DW}\right)$ & $32.2 \pm 1.35 \mathrm{c}$ & $52.6 \pm 2.87 \mathrm{~b}$ & $62.7 \pm 2.07 \mathrm{a}$ & $15.3 \pm 0.71 \mathrm{~d}$ & $32.1 \pm 1.58 \mathrm{c}$ \\
\hline & $\mathrm{T}-\mathrm{AOC}\left(\mathrm{U} \mathrm{g}^{-1} \mathrm{DW}\right)$ & $36.4 \pm 1.38 \mathrm{~b}$ & $50.9 \pm 1.74 \mathrm{a}$ & $51.7 \pm 1.91 \mathrm{a}$ & $16.0 \pm 0.55 \mathrm{c}$ & $35.4 \pm 2.23 b$ \\
\hline & $\mathrm{H}_{2} \mathrm{O}_{2}\left(\mu \mathrm{mol} \mathrm{g}{ }^{-1} \mathrm{DW}\right)$ & $14.9 \pm 1.22 b$ & $7.81 \pm 0.46 \mathrm{c}$ & $7.50 \pm 0.49 \mathrm{c}$ & $33.8 \pm 1.63 \mathrm{a}$ & $13.7 \pm 0.96 \mathrm{~b}$ \\
\hline
\end{tabular}

缩写同表 1。CK: 清水; T1: $10 \mathrm{nmol} \mathrm{L}{ }^{-1}$ 24-epiCS; T2: $10 \mathrm{nmol} \mathrm{L}^{-1}$ 28-homoBL; T3: $10 \mathrm{nmol} \mathrm{L}^{-1}$ brassinazole (BRZ, 芸薹素唑, BRs 合成 抑制剂); $\mathrm{T} 4: 10 \mathrm{nmol} \mathrm{L}{ }^{-1} 24-\mathrm{epiCS}+10 \mathrm{nmol} \mathrm{L}{ }^{-1} \mathrm{BRZ}$ 。数据为平均值土标准误, 同一排标以不同小写字母的值在处理间 0.05 水平上差 异显著 $(n=6)$ 。

Abbreviations are the same as those given in Table 1. CK: water control; T1: $10 \mathrm{nmol} \mathrm{L}{ }^{-1}$ 24-epiCS; T2: $10 \mathrm{nmol} \mathrm{L}^{-1}$ 28-homoBL; T3: 10 nmol L ${ }^{-1}$ brassinazole (BRZ, BRs synthesis inhibitor); T4: $10 \mathrm{nmol} \mathrm{L}^{-1} 24$-epiCS $+10 \mathrm{nmol} \mathrm{L}^{-1} \mathrm{BRZ}$. Data are means $\pm \mathrm{SE}$ with six independent replications. Values with different letters indicate significant differences at the 0.05 probability level within the same row $(n=6)$. 
表 3 外源化学调控物质对水稻颖花分化与退化、每穗粒数、饱粒率和粒重的影响

Table 3 Effects of applied chemical regulators on spikelet differentiation and degeneration, spikelet number per panicle, fully filled grains, and grain weight in rice

\begin{tabular}{lcccccc}
\hline $\begin{array}{c}\text { 品种 } \\
\text { Cultivar }\end{array}$ & $\begin{array}{c}\text { 处理 } \\
\text { Treatment }\end{array}$ & $\begin{array}{c}\text { 每穗颖花分化数 } \\
\text { Differentiated } \\
\text { spikelets per panicle }\end{array}$ & $\begin{array}{c}\text { 颖花退化率 } \\
\text { Spikelet } \\
\text { degeneration rate (\%) }\end{array}$ & $\begin{array}{c}\text { 每穗粒数 } \\
\text { Spikelets per } \\
\text { panicle }\end{array}$ & $\begin{array}{c}\text { 饱粒率 } \\
\text { Fully-filled } \\
\text { grains rate (\%) }\end{array}$ & $\begin{array}{c}\text { 千粒重 } \\
1000 \text {-grain weight } \\
(\mathrm{g})\end{array}$ \\
\hline 扬稻 6 号 & $\mathrm{CK}$ & $204 \pm 2.25 \mathrm{a}$ & $7.35 \pm 0.19 \mathrm{c}$ & $185 \pm 3.77 \mathrm{bc}$ & $88.2 \pm 1.69 \mathrm{~b}$ & $27.2 \pm 0.38 \mathrm{a}$ \\
YD-6 & $\mathrm{T} 1$ & $202 \pm 3.18 \mathrm{a}$ & $4.12 \pm 0.17 \mathrm{e}$ & $194 \pm 3.50 \mathrm{ab}$ & $92.3 \pm 1.81 \mathrm{a}$ & $26.8 \pm 0.40 \mathrm{a}$ \\
& $\mathrm{T} 2$ & $205 \pm 2.68 \mathrm{a}$ & $4.75 \pm 0.09 \mathrm{~d}$ & $196 \pm 3.83 \mathrm{a}$ & $92.2 \pm 1.80 \mathrm{a}$ & $26.6 \pm 0.52 \mathrm{a}$ \\
& $\mathrm{T} 3$ & $201 \pm 3.11 \mathrm{a}$ & $15.3 \pm 0.29 \mathrm{a}$ & $172 \pm 1.84 \mathrm{c}$ & $76.5 \pm 1.65 \mathrm{c}$ & $27.4 \pm 0.84 \mathrm{a}$ \\
& $\mathrm{T} 4$ & $207 \pm 3.49 \mathrm{a}$ & $8.03 \pm 0.15 \mathrm{~b}$ & $187 \pm 2.61 \mathrm{~d}$ & $88.3 \pm 1.35 \mathrm{~b}$ & $27.1 \pm 0.41 \mathrm{a}$ \\
角优 2640 & $\mathrm{CK}$ & $346 \pm 5.61 \mathrm{a}$ & $14.2 \pm 0.37 \mathrm{~b}$ & $292 \pm 3.94 \mathrm{~b}$ & $81.3 \pm 1.02 \mathrm{~b}$ & $24.9 \pm 0.40 \mathrm{ab}$ \\
YY-2640 & $\mathrm{T} 1$ & $348 \pm 6.63 \mathrm{a}$ & $8.92 \pm 0.25 \mathrm{c}$ & $313 \pm 5.40 \mathrm{a}$ & $84.2 \pm 1.37 \mathrm{a}$ & $24.4 \pm 0.29 \mathrm{ab}$ \\
& $\mathrm{T} 2$ & $343 \pm 4.95 \mathrm{a}$ & $9.07 \pm 0.17 \mathrm{c}$ & $310 \pm 4.56 \mathrm{a}$ & $84.4 \pm 1.45 \mathrm{a}$ & $24.4 \pm 0.30 \mathrm{ab}$ \\
& $\mathrm{T} 3$ & $349 \pm 4.42 \mathrm{a}$ & $28.4 \pm 0.87 \mathrm{a}$ & $241 \pm 2.97 \mathrm{c}$ & $65.3 \pm 1.04 \mathrm{c}$ & $25.3 \pm 0.60 \mathrm{a}$ \\
& $\mathrm{T} 4$ & $346 \pm 5.03 \mathrm{a}$ & $14.6 \pm 0.51 \mathrm{~b}$ & $288 \pm 4.41 \mathrm{~b}$ & $80.6 \pm 1.53 \mathrm{~b}$ & $25.0 \pm 0.37 \mathrm{ab}$ \\
\hline
\end{tabular}

缩写和处理同表 1 和表 2 。数据为平均值 \pm 标准误, 同列相同品种标以不同字母的值在 0.05 水平上差异显著 $(n=10)$ 。

Abbreviations and treatments are the same as those given in Tables 1 and 2. Data are means \pm SE with ten independent replications. Values with different letters indicate significant differences at the 0.05 probability level within the same column and cultivar $(n=10)$.

因)的控制，另一方面也说明氮素等外界营养因素 (外因)在调控水稻颖花退化过程中也发挥着重要作 用。本研究中颖花退化率在氮肥处理间差异较小的 重要原因可能是由于在氮肥处理中不仅保花肥的施 用量不同，前期的基肥、分藍肥、促花肥的施用量 均不同, 这导致不同的氮肥处理的有效穗数、分化 颖花数也相差很大，从而“稀释”了保花肥本身的效 应。此外, 在减数分裂期扬稻 6 号幼穗中的 BRs 含 量显著高于角优 2640 幼穗中的含量, 在同等施氮量 的条件下这一现象尤为明显, 这与两品种间颖花退 化率的差异状况相反(表 1 和图 2-A D)。从另一个方 面证明了 BRs 对水稻颖花退化的调控作用。表明稻 穗中具有较高的 BRs 水平可以作为减少水稻颖花退 化的一项重要生理指标, 通过品种选育或合理的栽 培措施增加稻穗的 BRs 生物合成与积累, 可有效减 少水稻颖花退化。这一发现对认识水稻颖花退化机 制、减少水稻颖花退化, 提高库容和产量潜力均具 有重要意义。BRs 介导施氮量对水稻颖花退化的遗 传与分子机制值得进一步深入探究。

\section{4 结论}

增加水稻减数分裂期幼穗中的 BRs 含量可以有 效降低颖花退化率。穗型较大的水稻品种比穗型较 小的品种需要施用更多的氮肥以增加减数分裂期幼 穗中 BRs 水平, 进而有效减少颖花退化。但氮肥施 用量过多导致减数分裂期稻穗含氮量过高会不利于
幼穗中 BRs 含量的增加, 进而增加颖花退化。当水 稻减数分裂期幼穗含氮量为 $1.25 \%$ 时, 可有效提高 水稻幼穗中的 BRs 水平, 从而减少颖花退化。BRs 通过调节减数分裂期稻穗的抗氧化能力介导不同施 氮量对水稻颖花退化的影响。

\section{References}

[1] FAOSTAT. FAO Statistical Databases, Food and Agriculture Organization (FAO) of the United Nations, Rome, 2016.

[2] Makino A. Photosynthesis, grain yield, and nitrogen utilization in rice and wheat. Plant Physiol, 2011, 155: 125-129.

[3] Peng S B, Tang Q Y, Zou Y B. Current status and challenges of rice production in China. Plant Prod Sci, 2009, 12: 3-8.

[4] 彭少兵. 对转型时期水稻生产的战略思考. 中国科学: 生命科 学, 2014, 44: 845-850.

Peng S B. Reflection on China's rice production strategies during the transition period. Sci Sin Vitae, 2014, 44: 845-850 (in Chinese with English abstract).

[5] Ashikari M, Sakakibara H, Lin S, Yamamoto T, Takashi T, Nishimura A, Angeles E R, Qian Q, Kitano H, Matsuoka M. Cytokinin oxidase regulates rice grain production. Science, 2005, 309: 741-745.

[6] Zhang W Y, Zhu K Y, Wang Z Q, Zhang H, Gu J F, Liu L J, Yang J C, Zhang J H. Brassinosteroids function in spikelet differentiation and degeneration in rice. $J$ Integr Plant Biol, 2019, 61: 943-963.

[7] Wang Z Q, Zhang W Y, Yang J C. Physiological mechanism underlying spikelet degeneration in rice. J Integr Agric, 2018, 17: 1475-1481.

[8] Zhang W Y, Chen Y J, Wang Z Q, Yang J C. Polyamines and ethylene in rice young panicles in response to soil drought during panicle differentiation. Plant Growth Regul, 2017, 82: 491-503. 
[9] Heng Y Q, Wu C Y, Long Y, Luo S, Ma J, Chen J, Liu J F, Zhang H, Ren Y L, Wang M, Tan J J, Zhu S S, Wang J L, Lei C, Zhang X, Guo X P, Wang H Y, Cheng Z J, Wan J M. OsALMT7 maintains panicle size and grain yield in rice by mediating malate transport. Plant Cell, 2018, 30: 889-906.

[10] Zhang W Y, Sheng J Y, Fu L D, Xu Y J, Xiong F, Wu Y F, Wang W L, Wang Z Q, Zhang J H, Yang J C. Brassinosteroids mediate the effect of soil-drying during meiosis on spikelet degeneration in rice. Environ Exp Bot, 2020, 169: 103887.

[11] Tang C J, Sun Y J, Xu H S, Yu S B. Identification of quantitative trait locus and epistatic interaction for degenerated spikelets on the top of panicle in rice. Plant Breed, 2011, 130: 177-184.

[12] Zhang D, Yuan Z. Molecular control of grass inflorescence development. Annu Rev Plant Biol, 2014, 65: 553-578.

[13] Lv B S, Tian H Y, Zhang F, Liu J J, Lu S H, Bai M Y, Li C Y, Ding $\mathrm{Z} \mathrm{J}$. Brassinosteroids regulate root growth by controlling reactive oxygen species homeostasis and dual effect on ethylene synthesis in Arabidopsis. PLoS Genet, 2018, 14: e1007144.

[14] Ye H X, Liu S Z, Tang B Y, Chen J N, Xie Z L, Nolan T M, Jiang H, Guo H Q, Lin H Y, Li L, Wang Y Q, Tong H N, Zhang M C, Chu C C, Li Z H, Aluru M, Aluru S, Schnable P S, Yin Y H. RD26 mediates crosstalk between drought and brassinosteroid signalling pathways. Nat Commun, 2017, 8: 14573.

[15] Zhang C, Bai M Y, Chong K. Brassinosteroid-mediated regulation of agronomic traits in rice. Plant Cell Rep, 2014, 33 683-696.

[16] Vriet G, Russinova E, Reuzeau C. From squalene to brassinolide: the steroid metabolic and signaling pathways across the plant kingdom. Mol Plant, 2013, 6: 1738-1757.

[17] Tong H, Liu L, Jin Y, Du L, Yin Y, Qian Q, Zhu L, Chu C. Dwarf and low-tillering acts as a direct downstream target of a GSK3/SHAGGY-Like kinase to mediate brassinosteroid responses in rice. Plant Cell, 2012, 24: 2562-2577.

[18] Sakamoto T, Morinaka Y, Inukai Y, Kitano H, Fujioka S. Auxin signal transcription factor regulates expression of the brassinosteroid receptor gene in rice. Plant J, 2013, 73: 676-688.

[19] Li D, Wang L, Wang M, Xu Y Y, Luo W, Liu Y J, Xu Z H, Li J, Chong K. Engineering OsBAK1 gene as a molecular tool to improve rice architecture for high yield. Plant Biotechnol J, 2009, 7: 791-806.

[20] Jiang W B, Huang H Y, Hu Y W, Zhu S W, Wang Z Y, Lin W H. Brassinosteroid regulates seed size and shape in Arabidopsis. Plant Physiol, 2013, 162: 1965-1977.

[21] Xin P, Yan J, Fan J, Chu J, Yan C. An improved simplified high-sensitivity quantification method for determining brassinosteroids in different tissues of rice and Arabidopsis. Plant Physiol, 2013, 162: 2056-2066.

[22] Zhang Z J, Chu G, Liu L J, Wang Z Q, Wang X M, Zhang H, Yang J C, Zhang J H. Mid-season nitrogen application strategies for rice varieties differing in panicle size. Field Crops Res, 2013, 150: 9-18

[23] Ali A, Xu P Z, Riaz A, Wu X J. Current advances in molecular mechanisms and physiological basis of panicle degeneration in rice. Int J Mol Sci, 2019, 20: 1613.
[24] 凌启鸿, 张洪程, 苏祖芳, 凌励. 稻作新理论. 北京: 科学出 版社, 1994. pp 98-120.

Ling Q H, Zhang H C, Su Z F, Ling L. New Theories in Rice Production. Beijing: Science Press, 1994. pp 98-120 (in Chinese)

[25] Namuco O S, O'Toole J C. Reproductive stage water-stress and sterility. Effect of stress during meiosis. Crop Sci, 1986, 26: 317-321.

[26] Ding J, Mao L J, Yuan B F, Feng Y Q. A selective pretreatment method for determination of endogenous active brassinosteroids in plant tissues: Double layered solid phase extraction combined with boronate affinity polymer monolith microextraction. Plant Methods, 2013, 9: 13.

[27] Chen M, Lu Y, Ma Q, Guo L, Feng Y Q. Boronate affinity monolith for highly selective enrichment of glycopeptides and glycoproteins. Analyst, 2009, 134: 2158-2164.

[28] Bajguz A, Tretyn A. The chemical characteristic and distribution of brassinosteroids in plants. Phytochemistry, 2003, 62: 1027-1046.

[29] Rao M, Lee H, Creelman R A, Mullet J E, Davis K R. Jasmonic acid signaling modulates ozone-induced hyper sensitive cell death. Plant Cell, 2000, 12: 1633-1646.

[30] Ling S, Chen C S, Wang Y, Sun X C, Lu Z H, Ouyang Y D, Yao J L. The mature anther-preferentially expressed genes are associated with pollen fertility, pollen germination and anther dehiscence in rice. BMC Genomics, 2015, 16: 101.

[31] Ding C Q, You J, Chen L, Wang S H, Ding Y F. Nitrogen fertilizer increases spikelet number per panicle by enhancing cytokinin synthesis in rice. Plant Cell Rep, 2014, 33: 363-371.

[32] Ding C Q, Wang Y, Chang Z Y, You S L, Liu Z H, Wang S H, Ding Y F. Comparative proteomic analysis reveals nitrogen fertilizer increases spikelet number per panicle in rice by repressing protein degradation and 14-3-3 Proteins. J Plant Growth Regul, 2016, 35: 744-754.

[33] Ghaley B B. Uptake and utilization of 5-split nitrogen topdressing in an improved and a traditional rice cultivar in the Bhutan Highlands. Exp Agric, 2012, 48: 536-550.

[34] Kamiji Y, Yoshida H, Palta J A, Sakuratani T, Shiraiwa T. N applications that increase plant $\mathrm{N}$ during panicle development are highly effective in increasing spikelet number in rice. Field Crops Res, 2011, 122: 242-247.

[35] Zhu X L, Liang W Q, Cui X, Chen M J, Yin C S, Luo Z J, Zhu J Y, Lucas W J, Wang Z Y, Zhang D B. Brassinosteroids promote development of rice pollen grains and seeds by triggering expression of carbon starved anther, a MYB domain protein. Plant $J$, 2015, 82: 570-581

[36] Zhang W Y, Sheng J Y, Xu Y J, Xiong F, Wu Y F, Wang W L, Wang Z Q, Yang J C, Zhang J H. Role of brassinosteroids in rice spikelet differentiation and degeneration under soil-drying during panicle development. BMC Plant Biol, 2019, 19: 409.

[37] Zhang W Y, Fu L D, Men C B, Men J X, Yao J Y, Sheng J Y, Xu Y J, Wang Z Q, Liu L J, Yang J C, Zhang J H. Response of brassinosteroids to nitrogen rates and their regulation on rice spikelet degeneration during meiosis. Food Energy Secur, 2020, 9: e201. 